\title{
Tendencias en el aprovechamiento de los residuos orgánicos biodegradables. Estudio cienciométrico
}

\section{Trends in the Use of Biodegradable Organic Waste. Scientometric Study}

\author{
Martha Cervantes Díaz', César Acevedo Argüello², \\ Teresa del Socorro Blanco Tirado ${ }^{3}$,Isabel Cristina Ocazionez
}

\section{Resumen}

El presente artículo se planteó con el propósito de identificar tendencias mundiales en la valorización de los residuos sólidos a partir de un estudio cienciométrico. Para lograrlo, se aplicó la herramienta de análisis cienciométrico de artículos, los cuales se obtuvieron de la base de datos Scopus (Elsevier, B.V., 2020) y se analizaron con el programa especializado de minería de texto VantagePoint (Search Technology, versión académica 12). Se recuperaron 483 artículos indexados en la base de datos, distribuidos durante el periodo del 2000 a la fecha (julio 2020); el año de mayor actividad fue el 2017 con 48 registros, seguido por el 2019 con 42 registros. El medio de divulgación científica más importante es Waste Management (32 artículos). En cuanto a la distribución geográfica a nivel mundial sobresale China con 90 publicaciones y en Latinoamérica, Brasil con 29. El interés del manejo de los residuos se orienta a procesos para la obtención de compost, biogás y biocombustibles, principalmente.

\footnotetext{
1 Grupo de Investigaciones Ambientales para el Desarrollo Sostenible, Facultad de Química Ambiental, Universidad Santo Tomás, seccional Bucaramanga, Bucaramanga, Santander. ORCID: (MCD) https://orcid.org/0000-0002-4427-6872; (ICO) https://orcid. org/0000-0001-9814-5045. Correo: martha.cervantes(Dustabuca.edu.co

2 Grupo de Investigación Espiral, Centro de Recursos para el Aprendizaje y la Investigación, CRAI-USTA, Universidad Santo Tomás, seccional Bucaramanga, Bucaramanga, Santander. ORCID: https://orcid.org/0000-0002-0979-4113

3 Grupo de Investigaciones USTAGRI, Facultad de Administración de Empresas Agropecuarias, Universidad Santo Tomás, seccional Bucaramanga, Bucaramanga, Santander. ORCID: https://orcid.org/0000-0002-0798-1302
} 


\section{Abstract}

This article aims to identify world trends in the recovery of solid waste from a scientometric study. The scientometric analysis tool was applied to articles obtained from the Scopus database (Elsevier, BV, 2020), then analyzed with the specialized text mining program VantagePoint (Search Technology, academic version 12). We retrieved 483 indexed articles published between 2000 to date (July 2020) from the database; the most significant activity took place in 2017 with 48 records, followed by 2019 with 42 records. The most important means of scientific dissemination is Waste Management (32 articles). Regarding geographical distribution worldwide, China stands out with 90 publications and, in Latin America, Brazil with 29. The interest in waste management is mainly oriented towards obtaining compost, biogas, and biofuels.

\section{Introducción}

En la actualidad, la gestión de los residuos sólidos representa uno de los principales desafíos de los sistemas económicos a nivel global, no solo por la cantidad en la que se producen en campos y ciudades, sino también por su naturaleza diversa; el peligro que representan para la salud humana y la estabilidad de los ecosistemas, y por las condiciones con las que cuenta cada región o gobierno en lo pertinente a tecnología, personal y recursos financieros para su recolección, transporte, almacenamiento y disposición final (Hussein y Mansour, 2018; OCDE, 2014; ONU, 1992).

Pero ¿qué se entiende por residuos sólidos? En el marco de la Declaración de Río sobre el Medio Ambiente en 1992, la Organización de las Naciones Unidas
(ONU, 1992) se empleó el término para incluir todo tipo de desecho:

todos los residuos domésticos y los desechos no peligrosos, como los desechos comerciales e institucionales, las basuras de la calle y los escombros de la construcción [...] en algunos países el sistema de gestión de desechos sólidos también se ocupa de los desechos humanos, tales como los excrementos, las cenizas de incineradores, el fango de fosas sépticas y el fango de instalaciones de tratamiento de aguas cloacales. (ONU, 1992)

$\mathrm{Su}$ estudio ha sido abordado desde diferentes perspectivas que van desde el análisis de su impacto sobre el agotamiento de los recursos de la naturaleza y la necesidad de gestionarlos para 
reducir la pérdida de sus servicios ecosistémicos (ONU, 1992), hasta la necesidad de identificar alternativas para su reincorporación a la economía (Gao et al., 2019; Soto-Paz et al., 2017; Torres et al., 2016).

De igual manera, hacen parte medular de los acuerdos internacionales para el cuidado del medio ambiente, como el plan de acción de las Naciones Unidas denominado Programa 21 (ONU, 1992), y en el contexto colombiano se consideran en políticas en las que se fomenta su aprovechamiento y reciclaje (DNP, 2008) y forma parte de métricas como los índices de oferta y utilización de residuos sólidos y productos residuales frente al producto interno bruto (PIB) como parte del Sistema de Contabilidad Ambiental y Económica Colombiano (DANE, 2016).

En Colombia, a partir de la Constitución Política de 1991 y los compromisos en los acuerdos internacionales como la Conferencia de Naciones Unidas sobre Medio Ambiente celebrada en Río de Janeiro en 1992, se formuló la Política Ambiental Colombiana mediante la Ley 99 de 1993, que sirve como base para un número de leyes, decretos, resoluciones y la creación de organismos e instituciones relacionados en diversos aspectos de la gestión de residuos sólidos (Castro-Buitrago et al., 2011).

Al revisar las políticas asociadas con la gestión de residuos sólidos, se destacan cuatro documentos asociados al cambio de percepción de los residuos: la Política para la Gestión Integral de Residuos de 1998; la Política Ambiental para la Gestión Integral de Residuos o Desechos Peligrosos de 2005; la Política de Producción y Consumo Sostenible de 2010, y la Política Nacional para la Gestión Integral de los Residuos Sólidos, Conpes 3874 de 2016.

La primera aborda dos aspectos fundamentales: el relacionado con la determinación de responsabilidades y estrategias para las entidades del Estado (municipios) encargadas del saneamiento y gestión apropiada de los residuos sólidos y el reconocimiento y consideración de los residuos generados por el sector privado. En cuanto a la naturaleza de los residuos, incluye los peligrosos y no peligrosos, dentro de los cuales reconoce la existencia de aprovechables y no aprovechables y la necesidad de dar manejo diferenciado a los aprovechables (Ministerio del Medio Ambiente, 1998).

A su vez, la segunda política identifica los actores generadores de residuos, 
su localización y contribución tanto en cantidad como en peligrosidad $\mathrm{y}$, en virtud del enorme riesgo que suponen para el medio ambiente y la salud humana, se concentra especialmente en plantear objetivos y metas con un horizonte de 12 años, hasta el 2018, y a dar los lineamientos sobre lo que debería ser el manejo de los residuos considerados como peligrosos, en un plan de acción con vigencia 2006-2010 (Ministerio de Ambiente, Vivienda y Desarrollo Territorial, 2005). Las dos políticas reconocen la importancia de la reducción en la generación de residuos desde la fuente.

En tercer lugar, la Política de Producción y Consumo Sostenible surge como nuevo paradigma de la gestión ambiental y asocia la gestión de los residuos con la gestión de las materias primas; incluye, además, la optimización del ciclo de vida de los productos, considera los resultados empresariales individuales frente a los obtenidos bajo un desempeño de una cadena productiva y reafirma la necesidad de un consumo responsable, entre otros. Destaca el análisis sobre la articulación de la política de producción y consumo sostenible con las demás políticas ambientales, entre las que se cuentan las relacionadas con la gestión de los residuos sólidos y la iden- tificación de sectores estratégicos en los que el manejo y reducción de residuos sólidos es clave (Colombia. Ministerio de Ambiente, Vivienda y Desarrollo Territorial, 2010).

Vale resaltar que según los resultados del desempeño ambiental de Colombia realizados por la Organización de Cooperación y Desarrollo Económicos (OCDE, 2014), en el país la mayoría de los desechos municipales no se separan desde la fuente y pasan directamente a los rellenos sanitarios, de los cuales $30 \%$ no cumple las normas ambientales. Situación que quizá pueda explicarse a partir de lo que esta institución señala como "la ausencia de un marco jurídico global para la gestión de residuos que sirva como guía de acción integral y coherente" (Castellanos, 2018).

La perspectiva del residuo sólido como fuente de ingreso queda consolidada en el documento Conpes 3874 del 2016, que contiene los lineamientos de la Política Nacional de Gestión de Residuos Sólidos. Su objetivo, impulsar el desarrollo de la Economía Circular hasta el 2030 en el país (DNP, 2016).

A raíz de la incorporación de Colombia a la OCDE, el cierre de ciclos de los materiales (economía circular) cobra importancia como generadora de acciones 
en pro de la mitigación del cambio climático. En el documento Conpes-ODS denominado Estrategia para la Implementación de los Objetivos de Desarrollo Sostenible (ODS) en Colombia, se definieron los indicadores para la medición del cumplimiento de las metas en materia de gestión de los residuos sólidos. Estos indicadores se incluyeron posteriormente en el eje de crecimiento verde: enfoque que implica un balance entre los propósitos económicos y los ambientales. Considerando lo anterior, se espera que durante el periodo comprendido entre el 2015 y 2030 se lleven las siguientes acciones en Colombia (Castellanos, 2018):

- Se reduzca la generación de residuos sólidos y productos residuales en $36,66 \%$ con relación al producto interno bruto (PIB) al pasar de 24.932 toneladas/billón de pesos a 15.788 toneladas/billón de pesos;

- Se incremente el porcentaje de residuos sólidos efectivamente aprovechados en $18 \%$, pasando de $17 \%$ al $35 \%$;

- Se aumente del $10,10 \%$ al $100 \%$ la eliminación de equipos y desechos de policlorobifenilos (PCB);

- Se incremente en $1.071,48 \%$ la gestión o aprovechamiento de los resi- duos de bombillas con mercurio, pasando de aprovechar o gestionar de 1.918 a 20.551 toneladas;

- Se incremente en $208 \%$ la tasa de reciclaje y nueva utilización de residuos sólidos generados; es decir, pasar de $8,59 \%$ a $17,89 \%$. (Castellanos, 2018)

Este trabajo tiene como propósito identificar las tendencias más relevantes en la gestión de los residuos sólidos orgánicos, para lo cual se empleó como herramienta metodológica la minería de texto de artículos científicos, comúnmente asociada a la cienciometría. Esta constituye el campo de estudio que tiene por objeto la investigación acerca del desarrollo del conocimiento científico y su estructura de comunicación, tal como lo afirma Van Raan (2019). Dicho campo de estudio se basa en datos cuantitativos provenientes, esencialmente, de los productos del proceso de investigación, desarrollo e innovación, tales como las publicaciones en revistas científicas y las patentes (Rousseau et al., 2018). Derivado de las técnicas cuantitativas aplicadas a la comunicación científica, como lo afirman Moral-Muñoz y colaboradores, surge el mapeo de la ciencia (science mapping) como una herramienta aplicada al 
descubrimiento de patrones y a la interpretación de la estructura y evolución de un campo de investigación (Moral-Muñoz et al., 2019). Este tipo de representaciones son útiles para reconocer dimensiones del desarrollo de un campo de investigación, tales como los asuntos estudiados con mayor énfasis, las relaciones con otros asuntos dentro del mismo campo de investigación, su desarrollo temporal y los actores clave (Petrovich, 2020).

\section{Materiales y métodos}

Se realizó un estudio cienciométrico sobre la investigación relacionada con el aprovechamiento de los residuos biodegradables, considerando el número de artículos indexados en Scopus (Elsevier, B.V., 2020), en el periodo 2000 - a la fecha de consulta (julio del 2020). Para el desarrollo de este ejercicio se estructuró la siguiente ecuación de búsqueda: TITLE-ABS-KEY (organic solid waste* $\mathrm{OR}$ organic solid residue* $\mathrm{OR}$ municipal organic waste*) AND DOCTYPE (ar) AND PUBYEAR > 1998. Se obtuvieron indicadores cienciométricos y matrices relacionales que permitieron definir las tendencias de investigación en este campo, empleando VantagePoint (Search Technology, versión académica 12.0), disponible a través del CRAI - Bi- blioteca de la Universidad Santo Tomás, seccional Bucaramanga. Este programa especializado permite hacer un análisis de minería de texto para identificar la evolución en el tiempo de las publicaciones, autores principales, los países e instituciones y aplicaciones por medio de matrices relacionales. Se empleó el VOSviewer (versión 1.6.15, 2020, Centre for Science and Technology Studies, Leiden University, The Netherlands) como programa de visualización para las matrices relacionales.

\section{Resultados}

A continuación, se presentan los resultados más relevantes de este ejercicio cienciométrico:

Áreas de conocimiento: de acuerdo con la ecuación de búsqueda anteriormente presentada, se identificaron 483 registros en Scopus que se relacionan con desechos de "residuos sólidos orgánicos" y "desechos orgánicos municipales". Las diferentes áreas de conocimiento en las cuales se aplican estas investigaciones se presentan en la figura 1. Las ciencias ambientales tienen un interés del $40 \%$, en tanto que el área de energía representa apenas $13 \%$, aplicaciones en el campo de la ingeniería química muestran un interés del 13\%, otras áreas representativas. 


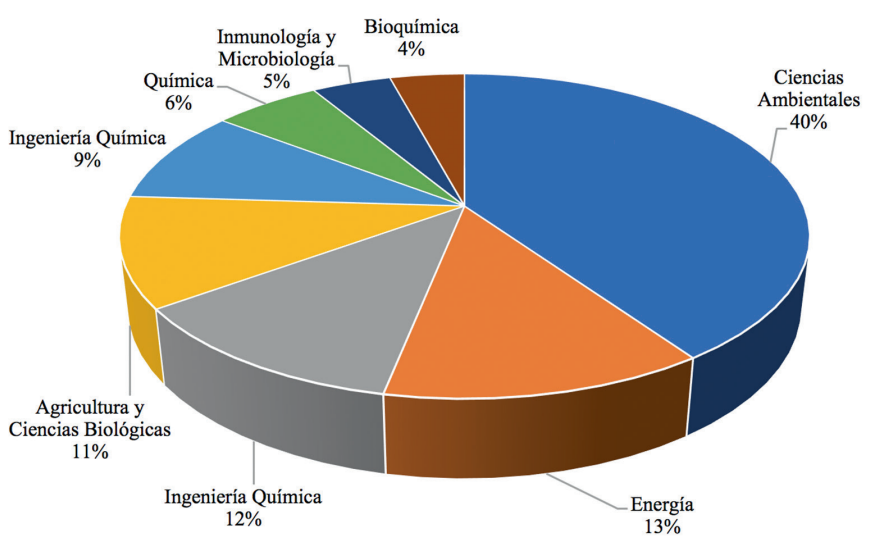

Figura 1. Distribución del interés de estudio de los residuos biodegradables de acuerdo con áreas de conocimiento

Fuente: Unidad de Bibliometría - CRAI Biblioteca Universidad Santo Tomás, seccional Bucaramanga. Scopus (Elsevier, B.V., 2020), VantagePoint (Search Technology, versión académica 12.0).

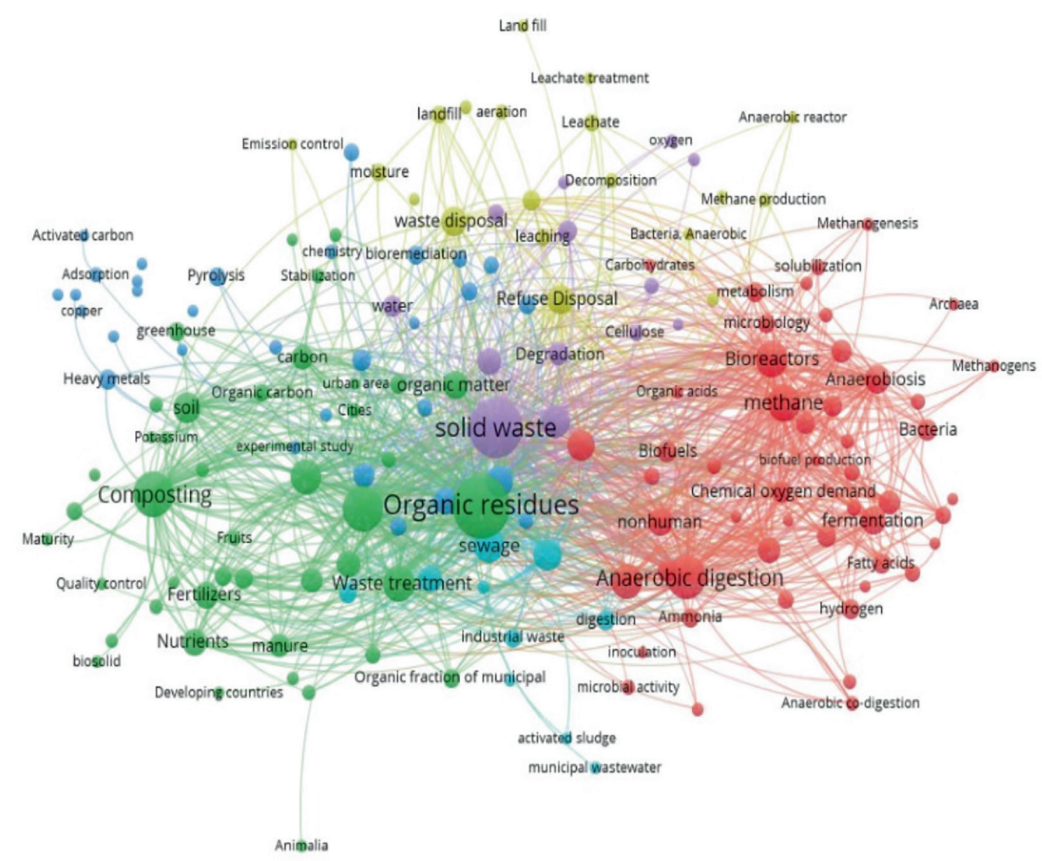

Figura 2. Mapa de co-ocurrencia de términos asociados con residuos biodegradables

Fuente: Unidad de Bibliometría - CRAl Biblioteca Universidad Santo Tomás, seccional Bucaramanga. Scopus (Elsevier, B.V., 2020), VantagePoint (Search Technology, versión académica 12.0), VoSviewer (versión 1.6.15, 2020). 
Mapa de co-ocurrencia de palabras clave: el mapa de red obtenido por medio de palabras clave presentes en 10 o más de los 483 registros estudiados, describen los temas, conceptos y método de investigación en el campo de los residuos sólidos orgánicos. En ese sentido, la representación de la figura 2 permite apreciar asuntos de investigación más fuertes gracias a la cantidad de publicaciones reportadas $y$, además, relacionadas más frecuentemente con otros descriptores temáticos. En la parte derecha de la figura se identifica el estudio de procesos anaerobios y asuntos asociados (anaerobic digestion, fermentation, methanogenesis, entre otros). A su vez, a la izquierda de la representación son visibles los procesos asociados al compostaje y la producción de fertilizante (composting, fertilizers). Además, en la parte superior de la representación se evidencian los estudios acerca de los rellenos sanitarios y la disposición de residuos (landfill, refuse disposal).

\section{Dinámica científica (artículos por año):} la actividad científica en esta temática se representa por la distribución del número de artículos por año (figura 3). Si bien no se observa una tendencia definida, sí se aprecia un interés constante en el tema, según la ecuación de búsqueda empleada. El año de mayor actividad fue el 2017 con 48 registros, seguido por el 2019 con 42 . En lo corrido del 2020, se registran a la fecha 36 documentos. Utilizando la Ley de Solla Price ${ }^{1}$ (De Solla Price, 1976), se calculó a partir del 2000 la tasa de crecimiento anual de los trabajos relacionados con desechos o residuos sólidos orgánicos (figura 3). Este índice fue de $18,35 \%$, con una correlación alta de los datos $\left(\mathrm{R}^{2}=0,9588\right)$.

Revistas científicas: los 482 artículos relacionados con los residuos sólidos orgánicos se publicaron en 223 revistas científicas, de las cuales 19 (8,5\%) contienen más de cuatro publicaciones sobre el tema. Las seis revistas más importantes en esta temática son Waste Management (32 artículos), Bioresource Technology (28 artículos), Water Science and Technology (14 artículos), Journal of Environmental Management (11 artículos), International Journal of Hydrogen Energy (10 artículos) y Journal of Cleaner Production (10 artículos), principalmente. Waste Management es una revista internacional orientada a la gestión integrada de residuos, ciencia y tecnología, tiene un factor de impacto de 5,448 y un puntaje de citaciones en el 2019 de 9,6.

1 La Ley de Price resulta de la observación del crecimiento del volumen de publicaciones científicas. Según se ha observado -con registros fiables desde el siglo xviii- que las publicaciones científicas tienen una tasa de crecimiento más o menos constante, valiendo ya sea para el conjunto de las publicaciones en todas las disciplinas consideradas como un todo, como para el análisis del crecimiento de cada campo por separado. 


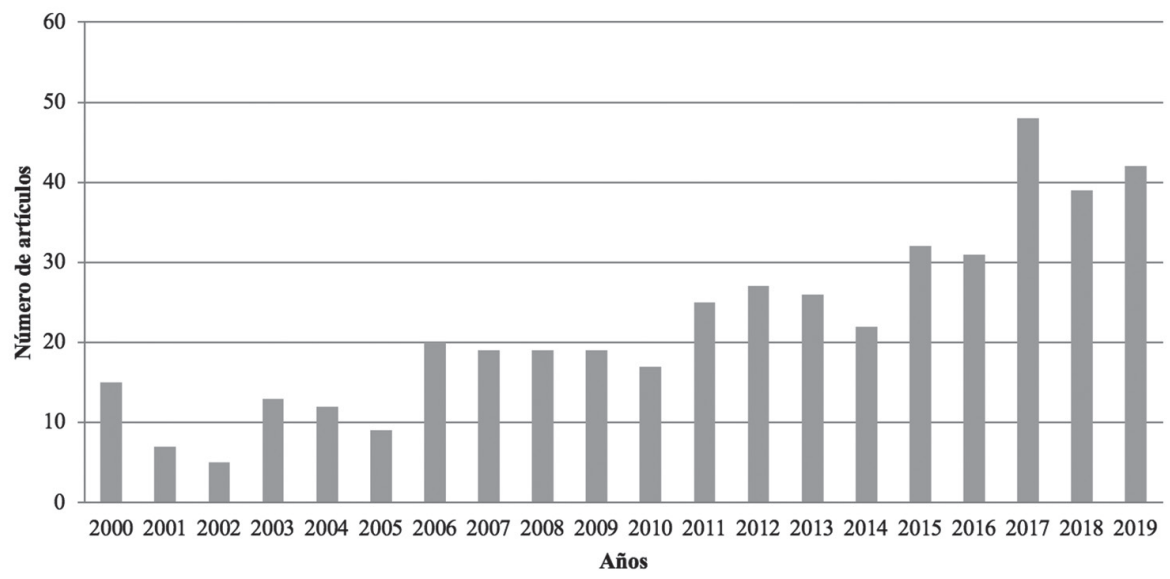

Figura 3. Dinámica científica y Ley de Solla Price del estudio de los residuos biodegradables Fuente: Unidad de Bibliometría - CRAl Biblioteca Universidad Santo Tomás, seccional Bucaramanga. Scopus (Elsevier, B.V., 2020), VantagePoint (Search Technology, versión académica 12.0).

Distribución por países: en cuanto a la distribución de las publicaciones por países a nivel mundial, se encontró que China presenta el mayor número de registros con 90 artículos, seguida por Japón con 47, India con 45, Estados Unidos con 32, Brasil y España con 30 documentos, entre otros. En países latinoamericanos, ya se mencionó a Brasil, México registra 27 publicaciones, Argentina con 6 , Colombia con 5, principalmente.

Matrices relacionales: se obtuvieron las matrices que relacionan los términos asociados con los residuos sólidos orgánicos con el interés de los países a nivel mundial y Latinoamérica (véase figura 4). Se puede apreciar que, para la mayoría de los países del mundo, especialmente China, la atención se orienta al manejo de los residuos orgánicos y el tratamiento de desechos, principalmente. En Latinoamérica, se observó la misma tendencia, pero en el caso de Brasil, México y Argentina, existe un interés en el manejo de residuos municipales.

En cuanto a productos o procesos de valorización de residuos o desechos sólidos orgánicos, se encuentra que mundialmente el interés está centrado en la obtención de compost, principalmente, seguido por el biogás, fertilizantes, biocombustibles, como se aprecia en la figura 5. En Latinoamérica, Brasil orienta sus investigaciones además del compostaje y el biogás, a la producción de energía, en especial de energías renovables. En el caso de Colombia, se orienta a compostaje y biogás. 

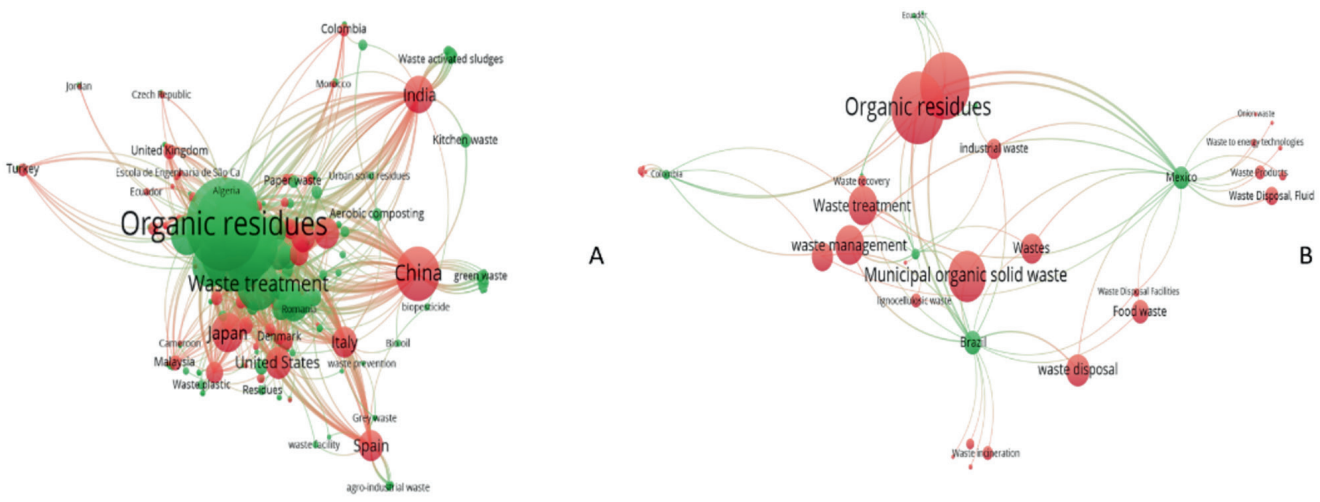

Figura 4. Relación de los términos asociados con el estudio de residuos biodegradables con países. A. A nivel mundial. B. Países latinoamericanos

Fuente: Unidad de Bibliometría - CRAl Biblioteca Universidad Santo Tomás, seccional Bucaramanga. Scopus (Elsevier, B.V., 2020), VantagePoint (Search Technology, versión académica 12.0), voSviewer (versión 1.6.15, 2020).

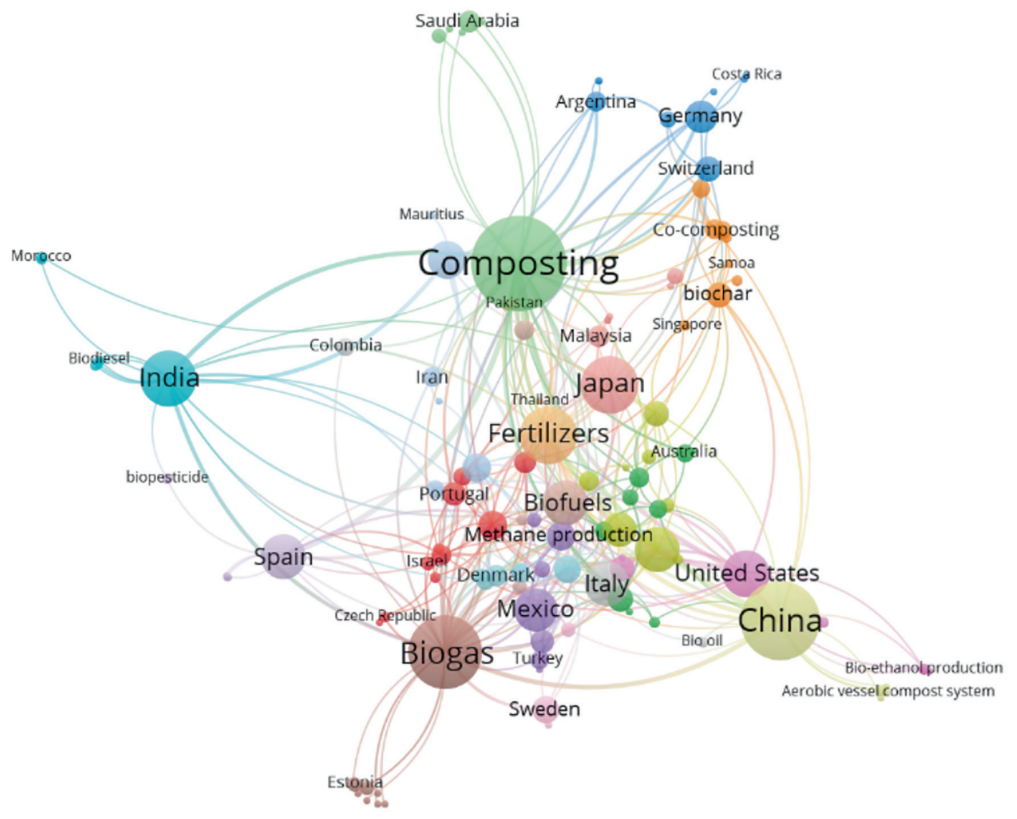

Figura 5. Relación de los términos asociados con valorización de residuos orgánicos a nivel mundial

Fuente: Unidad de Bibliometría - CRAl Biblioteca Universidad Santo Tomás, seccional Bucaramanga. Scopus (Elsevier, B.V., 2020), VantagePoint (Search Technology, versión académica 12.0), V0Sviewer (versión 1.6.15, 2020). 


\section{Conclusión}

Este trabajo muestra un panorama general sobre la investigación orientada al manejo de los desechos o residuos biodegradables. Si bien el número de registros recuperados con la ecuación de búsqueda planteada es poco, en casi 20 años se nota un creciente interés por este tema. En cuanto a la valorización, el interés se centra en el proceso de compostaje, estudios de diferentes procesos aerobios o anaerobios que aseguren la calidad del compost o biofertilizante. Otros productos de valorización es la obtención de biogás, biocombustibles, o generación de energías alternativas. Muy poco interés muestra el desarrollo del concepto de biorrefinerías para la obtención de compuestos de valor agregado como ácido láctico, flavonoides, entre otros, lo cual podría ser un nicho de desarrollo para la economía en Colombia.

\section{Agradecimientos}

Los autores expresan su agradecimiento a la Universidad Santo Tomás, seccional Bucaramanga, a través del CRAI Biblioteca por el apoyo a la realización de este trabajo.

\section{Referencias}

Castellanos, M. C. (2018). Propuesta de indicadores para medir los avances de la implementacion de una política de crecimien- to verde de largo plazo. 1-29. https://www. dnp.gov.co/Crecimiento-Verde/Documents/ ejes-tematicos/ODS/Indicadores Política Nacional de Crecimiento Verde final.pdf

Castro-Buitrago, E., Vásquez-Santamaría, J. y Jaramillo de los Ríos, L. (2011). La planeación urbana y la política de gestión de residuos sólidos en Medellín, cuestiones preliminares para un análisis jurídico y económico. Opinión Jurídica, 141-156.

DANE. (2016). Hoja metodológica de indicadores. Cuenta satélite ambiental. https:// www.dane.gov.co/files/investigaciones/pib/ ambientales/cuentas_ambientales/indicadores/cuenta-ambiental-y-economica-de-flujo-de-materiales/generacion-residuos-PIB/ hm-generacion-residuos-PIB.pdf

De Solla Price, D. (1976). A general theory of bibliometric and other cumulative advantage processes. Journal of the Association for information Science and Technology, 292-306.

DNP. (2008). Lineamientos y estrategias para fortalecer el servicio público de aseo en el marco de la gestión integral de residuos sólidos. Consejo Nacional de Politicas Económicas y Social de la República de Colombia, 53(9), 1689-1699. https://doi. org/10.1017/CBO9781107415324.004

DNP. (2016). Documento Conpes 3874. Politica Nacional para la Gestión Integral de Residuos Sólidos. Bogotá: Consejo Nacional de Política Económica y Social.

Gao, M., Zhang, M., Yue, S., Zheng, T., Gao, Z., Ma, X., \& Wang, Q. (2019). Global trends and future prospects of e-waste research: a bibliometric analysis. Environmental Science and Pollution Research, 26(17), 17809- 
17820. https://doi.org/10.1007/s11356019-05071-8

Hussein, A.-S., \& Mansour, M. S. M. (2018). Solid waste issue: Sources, composition, disposal, recycling, and valorization. Egyptian Journal of Petroleum, 27(4), 1275-1290. https://doi.org/10.1016/j. ejpe.2018.07.003

Ministerio de Vivienda, United States Agency for International Development (USAID), \& Iniciativa regional para el reciclaje inclusivo (IRR). (2015). Planes de gestión integral de residuos sólidos (PGIRS), 112. http://www. minvivienda.gov.co/Documents/ViceministerioAgua/PGIRS/PGIRS de Segunda Generación/Guía para la formulación, implementación, evaluación, seguimiento, control y actualización de los PGIRS.pdf

Ministerio de Ambiente, Vivienda y Desarrollo Territorial. (2010). Politica Nacional de Producción y Consumo Sostenible. https://www.corantioquia.gov.co/Site Assets/PDF/Gesti\%C3\%B3n\%20ambiental/Producci\%C3\%B3n\%20y\%20Consumo $\% 20$ Sostenible/P\%20PRODUCCION\%20CONSUMO.pdf

Moral-Muñoz, J. A., López-Herrera, A. G., Herrera-Viedma, E., \& Cobo, M. J. (2019). Science mapping analysis software tools: A review. En: W. Glänzel, H. F. Moed, U. Schmoch \& M. Thelwall (eds.), Springer Handbook of Science and Technology Indicators (pp. 159-185). Springer International Publishing. https://doi. org/10.1007/978-3-030-02511-3_7

OCDE. (2014). Evaluaciones de desempeño ambiental, 16. https://www.oecd.org/environment/country-reviews/Colombia $\mathrm{Hi}$ ghlights spanish web.pdf
ONU. (1992). Programa 21: Capítulo 21. Gestión ecológicamente racional de los desechos sólidos. Departamento de Asuntos Económicos y Sociales. División de Desarrollo Sostenible de las Naciones Unidas. https://www.un.org/spanish/esa/sustdev/ agenda21/agenda2 1 spchapter 21 .htm

Petrovich, E. (2020). Science mapping [Text]. https://www.isko.org/cyclo/science_mapping

Rousseau, R., Egghe, L., \& Guns, R. (2018). Scientific research and communication. En: Becoming Metric-Wise (pp. 11-35). E1sevier. https://doi.org/10.1016/B978-0-08102474-4.00002-9

Soto-Paz, J., Oviedo-Ocaña, R., Torres-Lozada, P., Marmolejo-Rebellón, L. F., \& Manyoma-Velásquez, P. C. (2017). Composting of biowaste: Research trends and relevance in developing countries. Dyna, 84(203), 334-342. https://doi.org/10.15446/dyna. v84n203.61549

Torres, S. C., Miranda, J. P. R., \& Ubaque, C. A. G. (2016). Optimization models of organic solid waste: Review article. International Journal of Environmental Protection, 6(1), 138-147. https://doi.org/10.5963/ ijep0601016

Van Raan, A. (2019). Measuring science: Basic principles and application of advanced bibliometrics. En: W. Glänzel, H. F. Moed, U. Schmoch \& M. Thelwall (eds.), Handbook of Science and Technology Indicators (pp. 237-280). Springer International Publishing. https://doi.org/10.1007/978-3030-02511-3_10 